\title{
Le projet Equipex NAOS : l'observation globale des océans Préparation de la nouvelle décennie d'Argo
}

\author{
Pierre-Yves Le Traon (Ifremer $\left.{ }^{1}\right)$, Fabrizio D'Ortenzio (UPMC/LOV ${ }^{2}$ ), \\ Marcel Babin (CNRS/UMI Takuvik ${ }^{3}$ ), Hervé Claustre (UPMC/LOV ${ }^{2}$ ), Sylvie Pouliquen (Ifremer ${ }^{1}$ ), \\ Serge Le Reste (Ifremer ${ }^{1}$ ), Virginie Thierry (IUEM/LPO ${ }^{4}$ ), Patrice Brault $\left(\mathrm{NKE}^{5}\right)$, \\ Michel Guigue $\left(\mathrm{CLS}^{6}\right)$, Marc Le Menn $\left(\mathrm{SHOM}^{7}\right)$. \\ ${ }^{1}$ Ifremer, Centre de Brest, BP70 29280 Plouzané \\ ${ }^{2}$ UPMC/LOV, Quai de La Darse, BP 806238 Villefranche sur Mer Cedex \\ ${ }^{3} \mathrm{CNRS} /$ UMI Takuvik, 1045, avenue de la Médecine, Université Laval, Québec, Canada \\ ${ }^{4}$ IUEM/LPO, Ifremer, Centre de Brest, BP70 29280 Plouzané \\ ${ }^{5}$ NKE, Rue Gutenberg, ZI de Kerandré 56700 Hennebont - France \\ ${ }^{6}$ CLS, 8-10 rue Hermès Parc Technologique du Canal 31520 Ramonville Saint-Agne \\ ${ }^{7}$ SHOM, 13 rue du Chatellier 29200 BREST
}

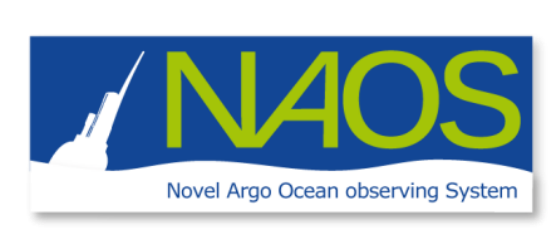

Résumé : NAOS (Novel Argo Ocean observing System) est l'un des 52 lauréats de l'appel à projets Equipex du programme d'investissements d'avenir. L'objectif de NAOS est de consolider la participation française et européenne au réseau international Argo de flotteurs profilants et d'anticiper les évolutions du réseau pour la prochaine décennie. L'enjeu est de mettre en place une véritable surveillance de l'ensemble des océans et de renforcer l'excellence française dans l'observation et la prévision de l'océan et du climat.

\section{NAOS Equipex project: Observing the World Ocean Preparing the next decade of Argo}

\begin{abstract}
NAOS (Novel Argo Ocean observing System) is one of the 52 projects selected as part of the Equipex call for proposals from the French programme d'investissements d'avenir. The overall objective of the project is to consolidate and improve the French and European contribution to the international Argo observing system and to prepare the next decade of Argo. The challenge is to set up an effective monitoring of the world ocean and to strengthen French leadership in ocean and climate research and prediction.
\end{abstract}
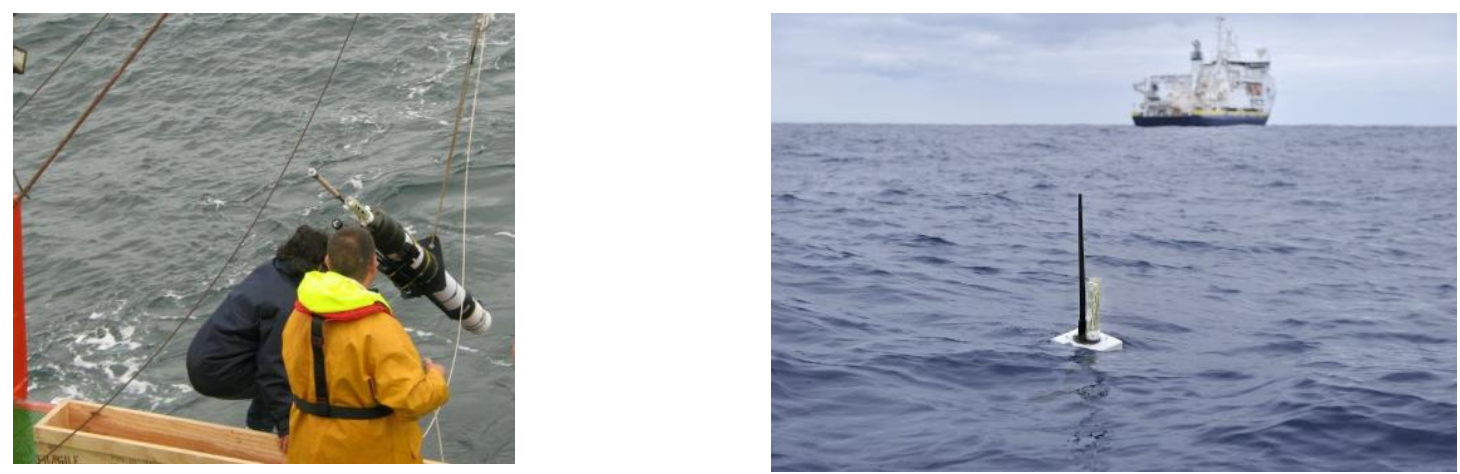


\section{L'observation des océans: la révolution Argo}

\section{I.1. Pourquoi observer les océans?}

Les océans stockent, transportent via les courants marins et échangent avec l'atmosphère d'énormes quantités de chaleur, d'eau et de gaz. Ces échanges ont une influence majeure sur le climat global et régional pour des échelles de temps allant de quelques jours (tempêtes et cyclones) à la saison (moussons) à plusieurs années (El Niño) et à plusieurs siècles (réchauffement climatique). Au cours des 50 dernières années, l'océan a ainsi absorbé 80 à $90 \%$ de l'excès de chaleur reçu par la planète dû à l'augmentation de la concentration des gaz à effet de serre dans l'atmosphère (Levitus et al, 2005).

Caractériser les effets du changement climatique, comprendre et prévoir l'évolution du climat de la planète nécessite une connaissance précise de l'océan, et des observations globales sur le long terme. C'est également indispensable pour les applications et services de l'océanographie opérationnelle.

\section{I.2. Le réseau international Argo}

Lancé en 2000 par la Commission océanographique intergouvernementale (COI) et l'Organisation météorologique mondiale (OMM), le programme Argo a pour objectif de développer un réseau global de 3000 flotteurs profilants autonomes mesurant en temps réel et tous les 10 jours la température et la salinité des 2000 premiers mètres de l'océan. Fin 2007, le projet a atteint son objectif initial avec 3000 flotteurs en opération. Argo est le fruit d'une coopération internationale remarquable dans laquelle plus de 30 pays participent directement à la mise en place du réseau. Chaque année 800 à 900 de ces instruments sont mis à l'eau, soit le nombre nécessaire pour maintenir un réseau de 3000 flotteurs actifs compte tenu de leur durée de vie estimée à 4 ans.

Argo est le premier réseau global et temps réel d'observation in-situ des océans. Les observations Argo ont une très forte complémentarité avec les observations des satellites (en particulier l'altimétrie satellitaire avec la série Jason).

Le réseau Argo est une composante essentielle du système mondial d'observation des océans et du climat permettant de :

- détecter la variabilité climatique aux échelles saisonnières et décennales, et observer à long terme le changement climatique dans les océans ;

- fournir des données essentielles pour contraindre des modèles d'analyse et de prévision océanique (GMES, Mercator Océan), afin d'initialiser des modèles couplés océan-atmosphère de prévision saisonnière et décennale et de valider les modèles climatiques ;

- fournir l'information nécessaire à l'étalonnage des capteurs et la validation des données satellitaires.

\section{I.3. Des premiers résultats extrêmement prometteurs}

Le réseau Argo n'est en place que depuis la fin de 2007 et ce n'est que sur la durée que ses bénéfices pourront être pleinement démontrés. En quelques années, Argo est cependant déjà devenu la plus importante source de données pour les chercheurs s'intéressant à l'étude de l'océan et de son rôle sur le climat. Freeland et al. (2010) donnent une synthèse des premiers résultats Argo. Les mesures Argo ont été utilisées pour mieux comprendre les variations du niveau moyen des mers (e.g. Cazenave et al., 2009) et pour analyser les variations de la circulation océanique à grande échelle (e.g. Roemmich et al., 2009). Elles ont permis de suivre des événements de convection profonde qui influent sur la circulation thermohaline et les flux méridiens de chaleur (Vage et al., 2009) (figure 1). Une contribution importante d'Argo a été d'améliorer nettement les estimations du stockage de chaleur par les océans (figure 2) (e. g. von Schuckmann et al., 2009 ; Trenberth, 2010 ; von Schuckmann et Le Traon, 2011). C'est un facteur déterminant pour estimer l'ampleur du réchauffement climatique, le bilan énergétique de la terre, et pour mieux comprendre les mécanismes de la montée du niveau moyen des mers. La comparaison des observations Argo aux climatologies passées a mis en évidence, par ailleurs, des variations à long terme de la salinité des océans ; celles-ci suggèrent une intensification du 
cycle de l'eau (Durack et Wijffels, 2010). L'utilisation des mesures Argo conjointement à celles altimétriques a enfin permis des avancées remarquables dans la représentation de l'océan nécessaire à la prévision océanique (e.g. Oke et al., 2009 ; Dombrowsky et al., 2009) et la prévision saisonnière (e.g. Balmaseda et al., 2009).

\section{I.4. Argo : une participation très active de la France}

La France est très active dans tous les aspects du programme Argo : développement de l'instrumentation (flotteurs Provor et Arvor et leurs dérivés), contribution au réseau par le déploiement de 60 à 80 flotteurs/an (soit environ $8 \%$ de l'effort international), centre de données (un des deux centres mondiaux de traitement se trouve au centre Coriolis de Brest), validation des mesures, recherche (circulation océanique, climat, biogéochimie) et océanographie opérationnelle (via Mercator Océan et GMES/MyOcean). La contribution française à Argo est coordonnée au sein de la structure inter-organismes Coriolis (composante in situ de l'océanographie opérationnelle) coordonnée par l'Ifremer et qui regroupe les principaux organismes français impliqués dans l'océanographie (CNES, CNRS/INSU, Ifremer, IPEV, IRD, Météo-France, SHOM). Le volet temps différé de Coriolis a été récemment labellisé comme Système d'Observation et d'Expérimentation pour la Recherche en Environnement (SOERE) qui fédère plusieurs systèmes d'observations (SO), dont le SO Argo France. Le SO Argo France regroupe les efforts des laboratoires français (notamment le LPO et le LOV) sur le traitement temps différé des données et la production de longues séries temporelles pour la communauté de recherche. Un financement ERC (European Research Council) a, par ailleurs, été attribué au LOV (H. Claustre) pour travailler sur le développement d'un flotteur biogéochimique.

La France coordonne également le développement d'Euro-Argo, la composante européenne d'Argo qui a été labellisé en 2006 dans la première feuille de route ESFRI (European Strategy Forum on Research Infrastructures) comme infrastructure de recherche européenne. Une phase préparatoire financée dans le cadre du $7^{\mathrm{e}}$ PCRD a démarré en janvier 2008 et s'est terminée en juin 2011. Les 12 pays partenaires (France, Allemagne, Grande-Bretagne, Italie, Espagne, Pays-Bas, Norvège, Irlande, Grèce, Portugal, Pologne, Bulgarie) se sont mis d'accord pour mettre en place une structure pérenne afin d'organiser sur la durée une contribution importante de l'Europe à Argo. La contribution visée est de 250 flotteurs/an, soit environ le quart du réseau global avec un échantillonnage densifié au niveau des mers régionales européennes.

La future Infrastructure de Recherche Euro-Argo comprendra une composante centrale et des composantes nationales distribuées (dont Argo France). La composante centrale aura une forme légale européenne et suivra le modèle proposé par la commission européenne (ERIC, European Research Infrastructure Consortium). La France va accueillir cette structure sur le site de l'Ifremer à Brest pour une phase initiale de 5 ans. Le dossier de mise en place de l'ERIC Euro-Argo entre dans sa dernière phase de validation au niveau des différents ministères et auprès de la commission européenne. L'objectif est sa mise en place au début de 2012.

\section{I.5. Les nouveaux défis pour Argo}

La dernière conférence Oceanobs'09 a défini les priorités de la communauté scientifique internationale pour l'observation globale des océans et pour Argo en particulier (Freeland et al., 2010 ; Claustre et al., 2010). La première priorité et le premier défi pour Argo est de maintenir le réseau actuel sur le long terme. La recherche sur le changement climatique a un besoin impératif d'observations globales sur le long terme et de très bonne qualité. Le second défi pour Argo est de faire évoluer le réseau pour répondre à de nouvelles questions scientifiques et élargir son domaine d'applications. L'observation des mers marginales est à la portée de la technologie actuelle, celles des zones polaires, complexe du fait de l'absence de possibilité de transmission directe en zone couverte de glace et des conditions climatiques extrêmes fait l'objet de travaux expérimentaux. Des évolutions majeures sont, en revanche, nécessaires pour l'observation de l'océan sous 2000 mètres et pour l'étude de la biogéochimie et des écosystèmes marins. Les mesures profondes sont nécessaires pour le suivi du climat et la prévision décennale et à plus long terme. Elles sont aussi importantes pour mieux comprendre les mécanismes de la montée du niveau moyen des mers. L'évolution d'Argo vers la biogéochimie est particulièrement prometteuse. Un groupe de travail international piloté par $\mathrm{H}$. 
Claustre a sélectionné les quatre premiers paramètres d'une version biogéochimique d'Argo : oxygène, nitrate, chlorophylle-a et carbone des particules. Cette sélection prend en compte à la fois la maturité technologique et les besoins scientifiques. L'oxygène est un paramètre clé pour caractériser le changement climatique et la vie marine. Le nitrate apporte, quant à lui, l'azote nécessaire à la formation de matière vivante et la chlorophylle-a est une mesure du phytoplancton produit par photosynthèse et premier maillon de la chaîne alimentaire. Le carbone des particules permet enfin de déterminer la quantité de matière vivante et morte.

Les mesures d'oxygène font déjà l'objet d'expériences pilotes et il est probable que d'ici quelques années une fraction importante des flotteurs Argo sera équipée de capteurs d'oxygène. Gruber et al. (2007) ont défini l'objectif d'un futur réseau Argo oxygène. Il s'agit de déterminer globalement, sur des échelles saisonnières à décennales, les variations de concentration en oxygène dans l'océan afin de:

- Surveiller la baisse attendue d'oxygène dans les océans (dégazage à cause du réchauffement et de la variation de solubilité associée, baisse de la ventilation des océans liée à l'augmentation de la stratification, aux modifications de la circulation méridienne et de la convection profonde) ;

- Suivre l'extension attendue des zones de minimum d'oxygène et de son impact sur les écosystèmes et la vie dans l'océan ;

- Quantifier l'amplitude et la variabilité de la production nette de la communauté ;

- Améliorer les estimations du stockage de $\mathrm{CO}_{2}$ anthropogénique dans l'océan ;

- Aider à l'interprétation des changements de circulation et de mélange océanique.

Des premières versions de flotteurs biogéochimiques, permettant des mesures plus complètes des paramètres biogéochimiques, ont été développées et testées au cours des années passées, notamment en France.

Ces évolutions du réseau Argo vont nécessiter des moyens nouveaux importants et une mobilisation forte de nouvelles communautés scientifiques. Sa mise en oeuvre doit donc être faite de façon progressive et coordonnée afin de ne pas affecter la mission de base Argo qu'il faut tout d'abord pérenniser. Si cette nouvelle phase est bien maîtrisée, il est fort probable que la deuxième décennie d'Argo apportera une nouvelle révolution dans l'observation des océans.

\section{Le projet Equipex NAOS}

\section{II.1. Objectifs du projet}

Maintenir le réseau Argo sur les prochaines décennies et assurer son évolution est un enjeu scientifique majeur tant pour les études climatiques que pour leurs implications environnementales et sociétales. L'objectif du projet Equipex NAOS (Novel Argo Ocean observing System) est de consolider et d'améliorer la contribution française et européenne à Argo et de préparer les prochains défis scientifiques pour Argo.

Le premier objectif de NAOS est de renforcer la contribution française au réseau international Argo par le déploiement de 10 à 15 flotteurs supplémentaires par an sur la période de 2012 à 2019 (110 flotteurs au total). Des développements technologiques innovants pour améliorer la fiabilité, la durée de vie, les économies d'énergie et les coûts seront menés. Grâce à NAOS, la contribution française au réseau international atteindra environ $10 \%$, soit un déploiement de l'ordre de 80 flotteurs/an.

Améliorer notre connaissance du rôle de l'océan sur le climat nécessite de nouveaux outils d'observation. Pour faire face aux échelles de temps très longues des changements climatiques, les masses d'eau profondes doivent être surveillées. Prendre en compte les impacts du changement climatique et les rétroactions implique de considérer les aspects biogéochimiques. Ces nouveaux défis scientifiques nécessitent une évolution des instruments Argo pour la prochaine décennie. NAOS va 
ainsi développer et valider la prochaine génération de flotteurs profilants Argo. 70 flotteurs de nouvelle génération seront déployés dans trois zones pilotes : la Méditerranée, l'Arctique et l'Atlantique nord.

\section{II.2. Partenariat}

Le projet NAOS résulte d'un partenariat fort entre l'Ifremer (coordinateur), l'UPMC (co-porteur), le CNRS, l'UBO/IUEM (PRES UEB), le SHOM et deux entreprises : CLS pour les aspects de télécommunications par satellite et la PME NKE qui est chargée de l'industrialisation et la commercialisation des flotteurs Argo. NAOS contribuera directement au développement du secteur économique via le partenariat avec NKE. L'objectif de NKE est d'accroître d'au moins un facteur deux ses parts de marché sur l'instrumentation Argo en Europe et à l'international.

\section{II.3. Détail des activités}

Le projet a démarré le $1^{\text {er }}$ juin 2011 et se terminera fin décembre 2019. Son budget total est de 8 Meuros dont 6 Meuros pour les phases de développement et les achats des séries de flotteurs et 2 Meuros pour soutenir les phases d'exploitation scientifique. NAOS est organisé autour de 5 workpackages principaux :

- WP1 : Consolidation de la contribution française à Argo (resp. Ifremer) ;

- WP2 : Développement de la nouvelle génération des flotteurs Argo (resp. Ifremer);

- WP3 : Flotteurs avec capteurs biogéochimiques en Méditerranée (resp. UPMC/LOV) ;

- WP4 : Flotteurs avec capteurs biogéochimiques en Arctique (resp. CNRS/UMI Takuvik);

- WP5 : Flotteurs profonds avec capteurs d'oxygène en Atlantique nord (resp. IUEM/LPO).

Le développement et le test des prototypes seront menés dans le WP2 de juin 2011 à juin 2014. Les achats de séries de flotteurs pour les WP1, 3, 4 et 5 s'étaleront de janvier 2012 à janvier 2016. Les premières séries pour le WP1 et le WP3 se baseront sur les modèles existants de flotteurs Argo (Provor et Arvor). Les commandes suivantes bénéficieront des avancées du WP2. Les achats pour les WP4 et WP5 se baseront exclusivement sur les nouveaux modèles de flotteurs développés dans le WP2.

Les activités des différents WPs sont détaillées ci-après.

\section{II.3.1. WP1 : Renforcer la contribution française à la mission globale Argo}

Grâce à NAOS, la France renforcera sa contribution à la mission de base Argo (mesure de température et de salinité tous les 10 jours de 0 à $2000 \mathrm{~m}$ ) en déployant 10 à 15 flotteurs supplémentaires par an de 2012 à 2019, complétant ainsi les 65 flotteurs financés par l'Ifremer et le SHOM. La stratégie de déploiement sera guidée par la contribution au réseau international et à l'infrastructure Euro-Argo visant à ensemencer en priorité les zones peu échantillonnées (notamment dans l'hémisphère sud) et à améliorer la couverture des mers européennes. Dès 2014, le WP1 devrait bénéficier des améliorations apportées aux profileurs dans le cadre du WP2.

\section{II.3.2. WP2: Développer la nouvelle génération des flotteurs Argo}

L'objectif est de préparer les évolutions instrumentales répondant aux besoins majeurs d'Argo pour les 10 années à venir. Les nouveaux flotteurs NAOS seront plus performants, plus "intelligents" et capables d'explorer l'océan "vert", les plus grandes profondeurs et les régions polaires. Les principales améliorations qui seront apportées sont listées ci-dessous :

- Réductions des coûts, fiabilité accrue, augmentation de la durée de vie pour les flotteurs standards (température et salinité) ;

- Évaluation d'un capteur de densité optique (NOSS) comme une alternative à terme aux mesures de conductivité pour la mesure de la salinité ;

- Nouvelles communications satellites (Argos-3, Iridium) : pour transmettre plus d'information, pour piloter le flotteur à distance et le reprogrammer (voie descendante) ;

- Développement d'un flotteur «souple» et «intelligent», disposant d'une architecture électronique et logicielle plus puissante. Cela facilitera l'ajout de nouveaux capteurs, offrira 
au vecteur une navigation plus versatile (schéma d'enchainement de phases élémentaires de descentes, dérives et remontées non figées), permettra la rétroaction de la «mesure » vers le vecteur (par exemple interrompre une remontée si de la glace de mer est détectée) ;

- Observations des plus grandes profondeurs (3500 m);

- Nouvelles capacités pour l'étude de la biogéochimie avec la possibilité d'ajouter une nouvelle suite de capteurs (oxygène, chlorophylle-a, nitrate, carbone) ;

- Mesures sous la glace : détection de la glace de mer (par méthode optique ou acoustique passive/active) avant la remontée, report de transmission des données si nécessaire.

Ce travail va s'organiser autour du développement et du test de 11 prototypes :

- 3 prototypes flotteurs Argo standard nouvelle génération;

- 2 prototypes flotteur Argo avec des nouvelles capacités de transmission Argos-3 ;

- 2 prototypes de flotteurs Argo $3500 \mathrm{~m}$;

- 2 prototypes embarquant un capteur Seabird et un capteur NOSS ;

- 2 prototypes de flotteur Argo biogéochimique arctique.

\section{II.3.3. WP3: Développer un premier réseau de flotteurs biogéochimiques en Méditerranée}

La communauté océanographique internationale a donné une priorité forte au passage progressif à la biogéochimie des réseaux d'observations in situ autonomes, pour l'instant principalement dédiés à la composante physique. La France est déjà fortement impliquée dans ces efforts pour Argo grâce au soutien de plusieurs organismes de recherche nationaux et européens. Cela se traduit déjà par :

- un développement technologique important qui a amené dans les années passées à la construction, au déploiement et à l'exploitation scientifique de flotteurs profileurs, qui, en plus des mesures Argo classiques, ont la capacité d'effectuer des mesures biogéochimiques ;

- une forte synergie entre le centre Coriolis et les équipes de recherche impliquées dans les services de qualification, de déploiement et de contrôle de qualité des données acquises par cette nouvelle génération de flotteurs profileurs biogéochimiques.

NAOS permettra de passer à l'étape suivante qui consistera à mettre en œuvre et maintenir un premier prototype de réseau de flotteurs biogéochimiques à l'échelle d'un bassin océanique. 33 flotteurs biogéochimiques de nouvelle génération seront ainsi déployés en mer méditerranée en 2013 et 2016. Ce nouveau réseau contribuera fortement aux actions scientifiques prévues en Méditerranée pour la prochaine décennie (Chantier Méditerranée, MERMEX, MOOSE).

\section{II.3.4. WP4: Observer l'impact du réchauffement climatique sur les écosystèmes en Arctique}

L'impact du réchauffement climatique sur les écosystèmes marins arctiques est indéniable et des perturbations majeures sont constatées au niveau de leurs propriétés les plus fondamentales : à savoir la couverture de glace, la lumière, les nutriments et la température. A cause de la fonte des glaces, les eaux de surface généralement abritées de la lumière naturelle, même en période estivale, se retrouvent éclairées 24 heures par jour. La pénétration du rayonnement solaire agit bien sûr sur le réchauffement de l'eau mais aussi sur la photosynthèse.

La marge de la banquise arctique est le lieu d'une production biologique intense au printemps et durant l'été. Des efflorescences de phytoplancton y sont souvent observées. Celles-ci pourraient représenter plus de la moitié de la production primaire pélagique arctique totale. Elles sont aussi probablement à l'origine d'une production secondaire et d'un flux de carbone organique particulaire vers les sédiments marins importants. Or, ce phénomène fondamental est encore mal compris. Quelles sont les conditions physiques et chimiques qui conditionnent leur développement? Quelles sont les espèces phytoplanctoniques majeures impliquées dans ces efflorescences ? Quelle est la dynamique de leur développement? Peut-on les prédire par modélisation?

Pour répondre à ces questions, NAOS étudiera les efflorescences de marge de glace observées chaque année en baie de Baffin. 13 flotteurs NAOS biogéochimiques Arctique seront déployés entre 2014 et 
2016. Un nombre au moins équivalent de flotteurs sera également déployé dans le cadre de la chaire d'excellence de M. Babin (UMI Takuvik).

II.3.5. WP5: Mettre en place un premier réseau de flotteurs profonds et oxygène en Atlantique Nord Le développement de flotteurs profonds capables de mesurer la concentration en oxygène dissous répond à deux grands enjeux scientifiques : avoir une meilleure estimation du rôle de l'océan dans l'élévation du niveau de la mer en accédant à la composante thermique profonde (en dessous de 2000 $\mathrm{m}$ ) et mettre en place une surveillance globale de la concentration en oxygène dans l'océan.

24 flotteurs NAOS équipés de capteurs d'oxygène et capables de faire des mesures jusqu'à $3500 \mathrm{~m}$ seront déployés dans l'Atlantique nord entre 2014 et 2016. Les objectifs scientifiques particuliers sont l'étude de la formation des masses d'eaux modales dans le gyre subpolaire, l'étude de la convection profonde et de la ventilation de l'océan intérieur et la détermination du taux de stockage, du transport d'oxygène et des échanges avec les moyennes latitudes.

\section{Conclusion}

L'observation des océans est devenue une nécessité absolue. Les enjeux sont considérables : caractérisation du changement climatique dans l'océan (réchauffement de l'océan superficiel et profond, évolution de la salinité en réponse à des variations du cycle hydrologique, fonte des glaces, niveau de la mer, capacité d'absorption du $\mathrm{CO}_{2}$, acidification, évolution des écosystèmes), meilleure compréhension du rôle de l'océan et des couplages physique et biologie, prévision saisonnière et décennale, océanographie opérationnelle...

Cette observation est organisée autour d'un système global international s'appuyant à la fois sur des observations satellitaires et des mesures in situ, puis sur une intégration via la modélisation et l'assimilation de données. Argo en est une composante essentielle et une des avancées majeures de l'océanographie des 20 dernières années. Les nouveaux défis pour Argo aujourd'hui sont de pérenniser le réseau et de le faire évoluer. Le projet NAOS répond directement à ces deux attentes et va permettre aux chercheurs français de maintenir une position de premier plan dans l'observation des océans, l'océanographie opérationnelle et l'étude du changement climatique. C'est une opportunité unique pour la communauté scientifique française. Via le partenariat avec NKE, NAOS va parallèlement permettre de développer l'offre industrielle des flotteurs français au niveau européen et international.

\section{Remerciements}

Le projet NAOS bénéficie d'une aide de l'Etat au titre du programme "Investissements d'avenir ». Cette aide gérée par l'Agence nationale de la recherche porte la référence ANR-10-EQPX-40.

\section{Références}

- Balmaseda, M., et D. Anderson, 2009 : Impact of initialization strategies and observations on seasonal forecast skill. Geoph. Res. Lett., 36, L01701.

- Cazenave, A., K. Dominh, S. Guinehut, E. Berthier, W. Llovel, G. Ramillien, M. Ablain et G. Larnicol, 2009 : Sea level budget over 2003-2008: A reevaluation from GRACE space gravimetry, satellite altimetry and Argo. Global and Planetary Change, 65, 83-88.

- Claustre, H., D. Antoine, L. Boehme, E. Boss, F. D'Ortenzio, O. Fanton D'Andon, C. Guinet, N. Gruber, N. O. Handegard, M. Hood, K. Johnson, A. Körtzinger, R. Lampitt, P.-Y. Le Traon, C. Lequéré, M. Lewis., M. J. Perry, T. Platt, D. Roemmich, S. Sathyendranath, P. 
Testor, U. Send et J. Yoder, 2010a : Guidelines Towards an Integrated Ocean Observation System for Ecosystems and Biogeochemical Cycles. Proceedings of the OceanObs'09: Sustained Ocean Observations and Information for Society Conference (Vol. 1), Venice, Italy, 21-25 September 2009, Hall, J., Harrison D. E. and Stammer, D., Eds., ESA Publication WPP306.

- Dombrowsky, E., L. Bertino, G. B. Brassington, E. P. Chassignet, F. Davidson, H. E. Hurlburt, M. Kamachi, T. Lee, M. J. Martin, S. Mei et M. Tonani, 2009 : GODAE Systems in Operation. Oceanography, 22(3), 80-95.

- Durack, P .J., et S. E. Wijffels, 2010 : Fifty-year trends in global ocean salinities and their relationship to broad-scale warming. J. Climate, 23, 4342-4362.

- Freeland, H. J., D. Roemmich, S. L. Garzoli, P.-Y. Le Traon, M. Ravichandran, S. Riser, V. Thierry, S. Wijffels, M. Belbeoch, J. Gould, F. Grant, M. Ignazewski, B. King, B. Klein, K. A. Mork, B. Owens, S. Pouliquen, A. Sterl, T. Suga, M.-S. Suk, P. Sutton, A. Troisi, P. J. VelezBelchi et J. Xu, 2010 : Argo - A Decade of Progress. Proceedings of OceanObs'09: Sustained Ocean Observations and Information for Society (Vol. 2), Venice, Italy, 21-25 September 2009, Hall, J., Harrison D.E. \& Stammer, D., Eds., ESA Publication WPP-306.

- Gruber, N., Doney S. C., S. R. Emerson, D. Gilbert, T. Kobayashi, A. Körtzinger, G. C. Johnson, K. S. Johnson, S. C. Riser et O. Ulloa, 2007 : The Argo-oxygen program. A white paper to promote the addition of oxygen sensors to the international Argo float program.

- Harrison, D. E., et D. M. Legler, 2010 : Saltier, Hotter, More Acidic, and Less Diverse? Observing the Future Ocean. EOS Trans. AGU, 91(3), 23.

- Levitus, S., J. Antonov et T. Boyer, 2005 : Warming of the world ocean, 1955-2003. Geophys. Res. Lett., 32, L02604, doi:10.1029/2004GL021592.

- Oke, P. R., M. A. Balmaseda, M. Benkiran, J. A. Cummings, Y. Fujii, S. Guinehut, G. Larnicol, P.-Y. Le Traon, M. J. Martin, E. Dombrowsky, 2009 : Observing System Evaluations using GODAE systems. Oceanography, 22(3), 144-153.

- Roemmich, D., and the Argo Steering Team, 2009 : Argo: the challenge of continuing 10 years of progress. Oceanography, 22(3), 46-55, doi:10.5670/oceanog.2009.65 .

- Trenberth, K. E., 2010 : The ocean is warming, isn't it? Nature, 465, 304.

- Vage, K., R. S. Pickart, V. Thierry, G. Reverdin, C. M. Lee, B. Petrie, T. A. Agnew, A. Wong et M. H. Ribergaard, 2009 : Surprising return of deep convection to the subpolar North Atlantic Ocean in winter 2007-2008. Nature Geoscience, 2, 67-72.

- von Schuckmann, K., F. Gaillard et P. Y. Le Traon, 2009 : Global hydrographic variability patterns during 2003-2008. J. Geoph. Res. Oceans, 114, 17.

- von Schuckmann, K., et P. Y. Le Traon, 2011 : Global ocean climate indices derived from Argo data from 2005 to 2010. Ocean Science (en révision). 


\section{Figures}

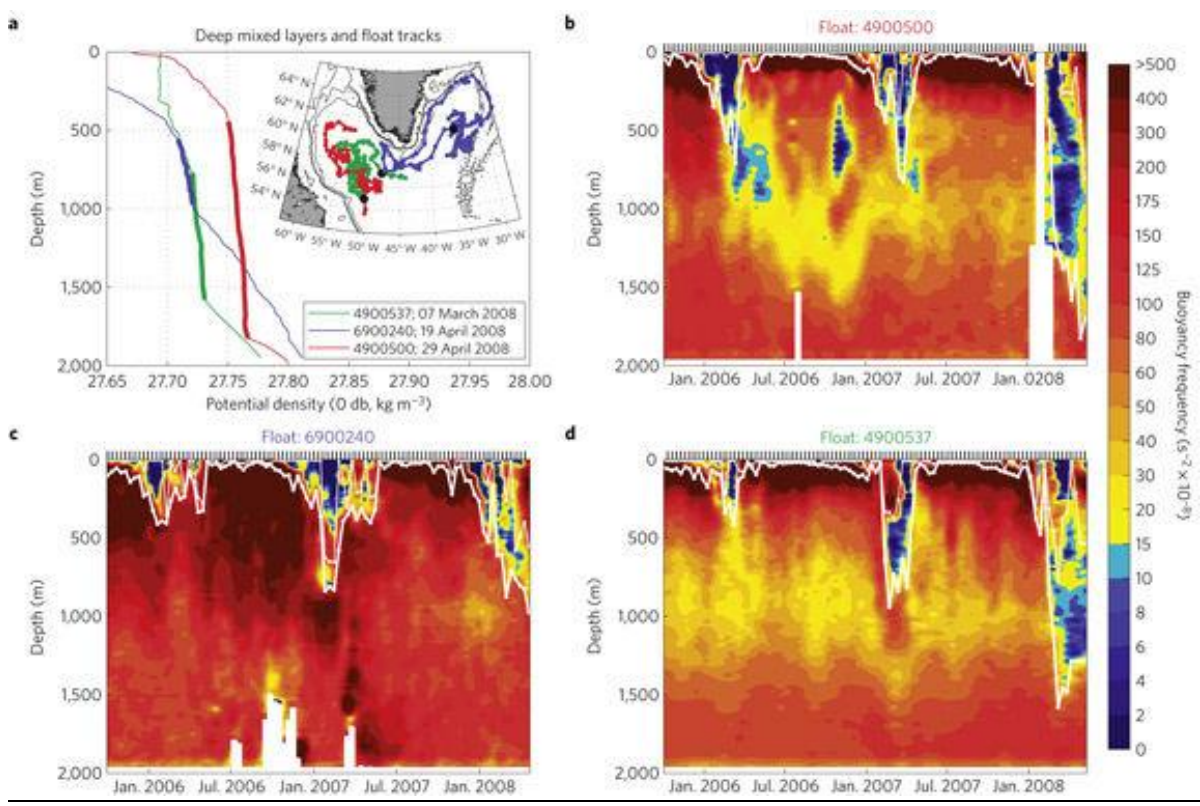

Figure 1 : Les observations de 3 flotteurs Argo en mer du Labrabor montrent un retour de la convection profonde durant l'hiver 2007-2008 (Vage et al., 2009).

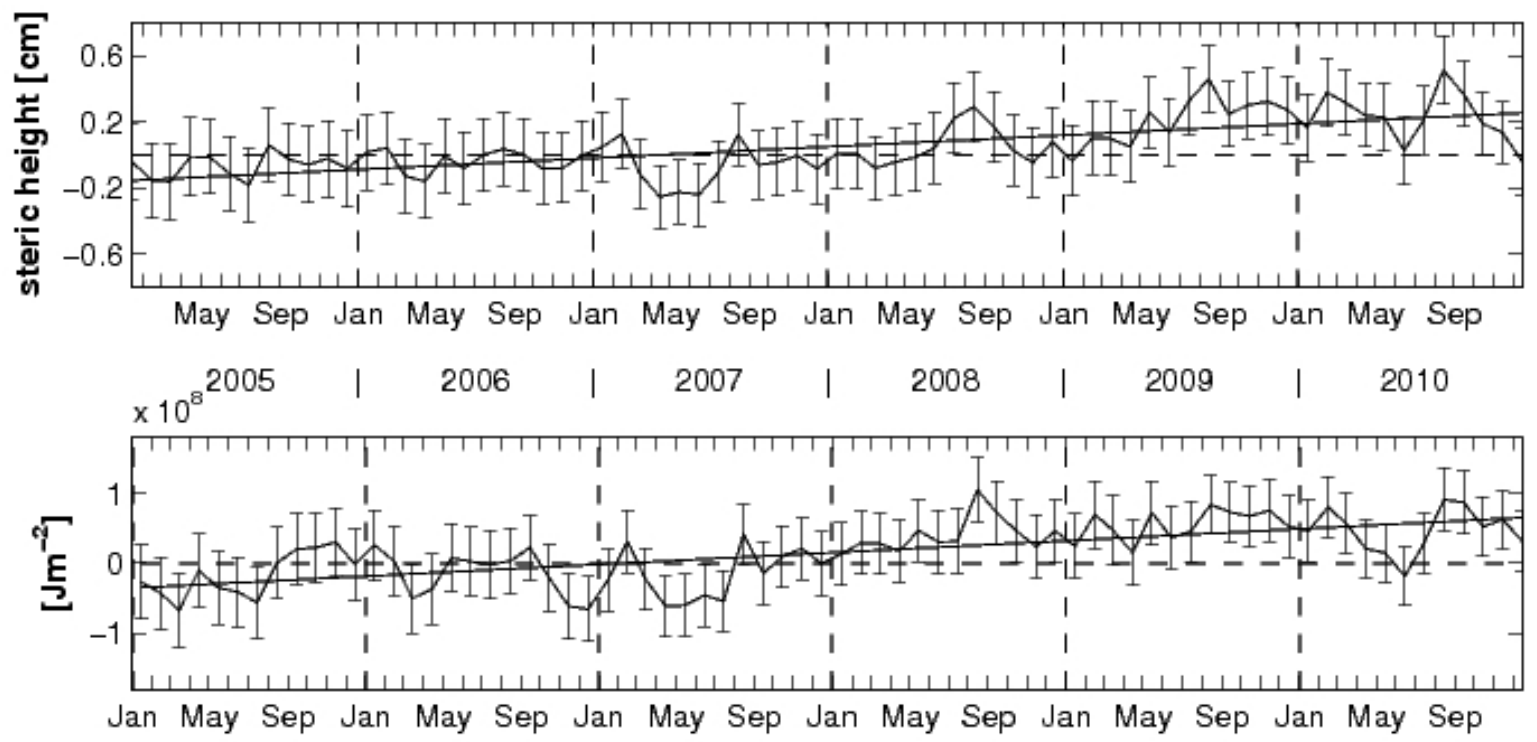

Figure 2: Contenu moyen de chaleur stockée dans les océans déduit des données Argo (2005-2010) et variations associées du niveau moyen des mers (von Schuckmann et al., 2009 ; von Schuckmann et Le Traon, 2011). 


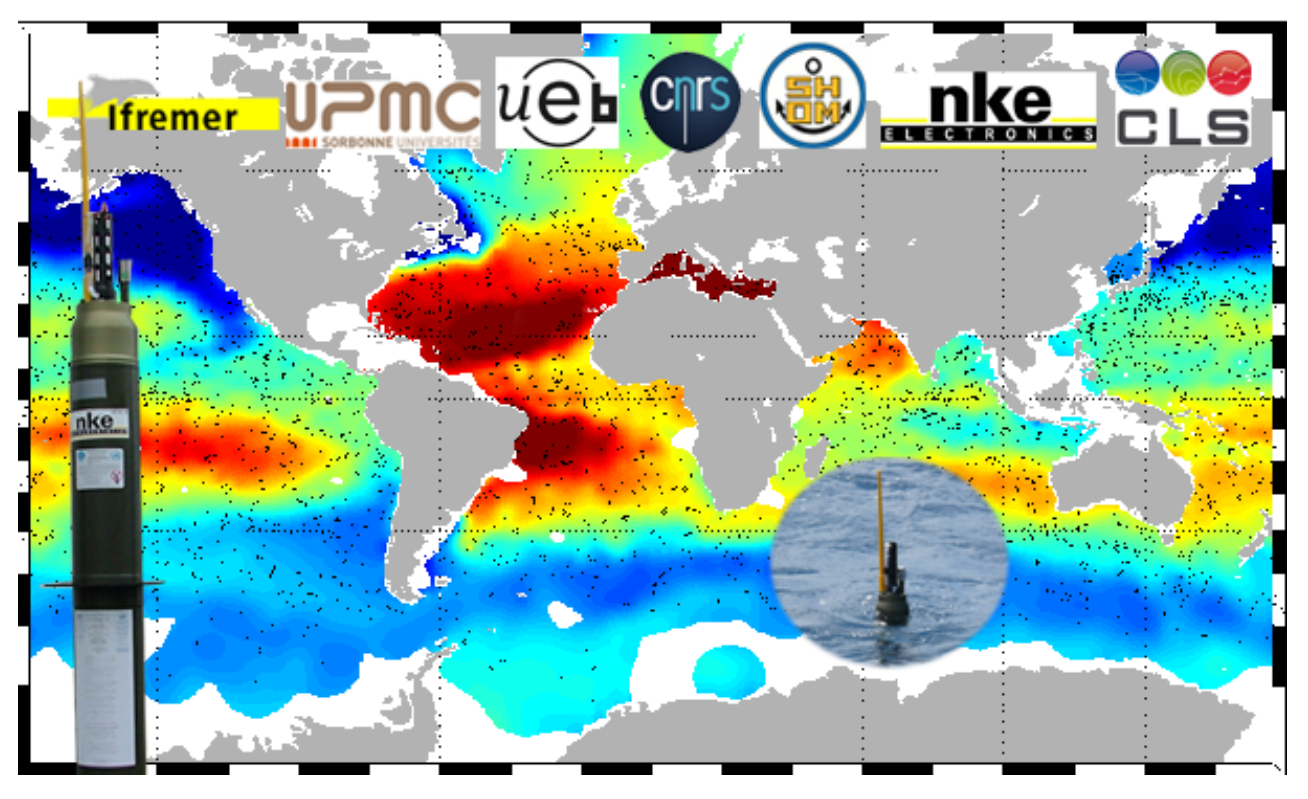

Figure 3: Partenariat NAOS. 